\title{
Bivariate and Bilateral Gamma Distributions
}

\author{
Yusra A. Tashkandy ${ }^{1}$, Maha A. Omair ${ }^{2} \&$ Abdulhamid A. Alzaid ${ }^{3}$ \\ 1,2,3 Department of Statistics and Operations Research, College of Sciences, King Saud University, Riyadh, Kingdom of \\ Saudi Arabia \\ Correspondence: Maha A. Omair, Department of Statistics and Operations Research, College of Sciences, King Saud \\ University, Riyadh, Kingdom of Saudi Arabia. E-mail: maomair@ksu.edu.sa
}

Received:December 8, 2017 Accepted:December 26, 2017 Online Published: February 15, 2018

doi:10.5539/ijsp.v7n2p66

URL: https://doi.org/10.5539/ijsp.v7n2p66

\begin{abstract}
This article review some known bivariate and bilateral (difference) gamma distributions. Some properties, advantages and limitations are pointed out. Two new bivariate gamma distributions using self-decomposability property are introduced. The corresponding bilateral gamma distributions are derived.
\end{abstract}

Keywords: bilateral distribution, bivariate distribution, hypergeometric function, Whittaker function

\section{Introduction}

In many real-life applications, more than one variable are collected on each individual. Therefore, multivariate distributions are needed to model and explain these variables where strong dependencies are possible. Bivariate distributions can be constructed by different methods. The simplest way is the reduction method. Copulas allow creating different bivariate distributions with different association parameters. Hutchinson and Lai (1990) discussed some of these distributions. Also, some bivariate distributions using compound distributions are defined, see for example Hutchinson (1981).

Bivariate gamma distributions are derived in the literature and commonly used to analyze skewed data. Balakrishnan and Lai (2009) listed different form of bivariate gamma distributions. Some of these have non-gamma marginal distributions such as Becker and Roux (1981) and Malik and Trudel (1985) who generalized Kibble bivariate gamma distribution. In this paper, we will focus only on bivariate gamma distributions whose marginals are gamma distributions.

In recent years, many researchers have paid attention to the difference of two random variables belonging to the same family of distributions such as differences of Poisson and gamma. Here we are interested in the difference of gamma variables. Cheng and Berger (2003) have defined the difference of two sums of independent generalized gamma random variables. Holm and Alouini (2004) have introduced the difference of two independent gamma random variables for the case of equal shape parameters. They proved that it followed the second of MckKay's (1932) distribution and computed the moments and the cumulative distribution functions. They also discussed the distribution of the difference between two correlated gamma random variables. The distribution of the difference of two independent sums of a finite number of gamma random variables with different rate parameters and integer shape parameters was obtained by Coellho and Mexia (2007).

Recently, Küchler and Tappe (2008a) considered the distribution of the difference between two independent gamma random variables with different shapes and scales parameters and refered to it as bilateral gamma distribution. They studied some of its properties such as moments, self-decomposability, and closeness under convolution. Additional properties especially those related to the shape of the density can be found in Küchler and Tappe (2008b). Küchler and Tappe (2008b) also gave representations of the density in terms of the Whittaker function.

Bilateral gamma distributions can be used to model real life applications. For example, Holm and Alouini (2004) and Coellho and Mexia (2007) used two special cases of bilateral gamma distributions to compute the outage probability. Küchler and Tappe (2008a) used the distribution to model stock prices. Küchler and Tappe (2008b) applied the bilateral gamma distribution to model the financial market fluctuation. Option pricing with bilateral gamma distribution in continuous time models had been considered by Küchler and Tappe (2009). Bellini and Mercuri (2012) considered modeling option pricing using bilateral gamma GARCH type model.

As there is more than one definition of the bivariate gamma distribution, it would be of interest to discuss their corresponding difference distributions. The aim of the paper is to review 7 different form of the bivariate gamma distributions and define two new bivariate gamma distributions based on self-decomposability property. And examine the 
similarity and dissimilarity of the resulting difference distributions. The article is organized as follows. Some bivariate gamma distributions are reviewed in Section 2. In Section 3, we introduce two new bivariate gamma distributions. We discussed the corresponding gamma difference (bilateral) distribution and derived other new bilateral gamma distributions in Section 4.

Throughout we denote by $\Gamma(\alpha, \lambda)$ to the gamma distribution with probability density function

$$
g(x)=\frac{1}{\lambda \Gamma(\alpha)}(x / \lambda)^{\alpha-1} e^{-x / \lambda}, x>0 .
$$

As usual, we call $\alpha$ the shape parameter and $\lambda$ the scale parameter.

The calculations involve several special functions from Gradshteyn and Ryzhik (2007) (G-R), and for ease of references we list them here with the corresponding pages

The Pochhammer symbol is defined by [G-R, p. xliii]

$$
(e)_{k}=e(e+1) \ldots(e+k-1)=\frac{\Gamma(e+k)}{\Gamma(e)} .
$$

The Gauss' hypergeometric function is defined by [G-R, p. 1005]

$$
{ }_{2} F_{1}(a, b ; c ; z)=\sum_{n=0}^{\infty} \frac{(a)_{(n)}(b)_{(n)} z^{n}}{(c)_{(n)} n !} .
$$

This series converges for $|z|<1$.

The integral representation of (1) is

$$
{ }_{2} \mathrm{~F}_{1}(\mathrm{a}, \mathrm{b} ; \mathrm{c} ; \mathrm{z})=\frac{1}{\mathrm{~B}(\mathrm{~b}, \mathrm{c}-\mathrm{b})} \int_{0}^{1} \mathrm{t}^{\mathrm{b}-1}(1-\mathrm{t})^{\mathrm{c}-\mathrm{b}-1}(1-\mathrm{zt})^{-\mathrm{a}} \mathrm{dt}, \text { for } \mathrm{c}>b>0 \text {. }
$$

The confluent hypergeometric function

$$
{ }_{1} F_{1}(a ; b ; z)=\sum_{n=0}^{\infty} \frac{(a)_{n} z^{n}}{(b)_{n} n !}
$$

This series converges for the values of $|z|<1$.

The integral representation of (3) is

$$
{ }_{1} \mathrm{~F}_{1}(\mathrm{a} ; \mathrm{b} ; \mathrm{z})=\frac{2^{1-\mathrm{b}} \mathrm{e}^{\frac{1}{2} \mathrm{z}}}{\mathrm{B}(\mathrm{a}, \mathrm{b}-\mathrm{a})} \int_{-1}^{1}(1-\mathrm{t})^{\mathrm{b}-\mathrm{a}-1}(1+\mathrm{t})^{\mathrm{a}-1} \mathrm{e}^{\frac{1}{2} \mathrm{zt}} \mathrm{dt} ; \text { for } 0<a<b .
$$

The Whittaker function is defined by [G-R, p. 1024]

$$
W_{\alpha, \beta}(z)=\frac{z^{\alpha} e^{-\frac{z}{2}}}{\Gamma\left(\beta-\alpha+\frac{1}{2}\right)} \int_{0}^{\infty} e^{-t} t^{\beta-\alpha-\frac{1}{2}}\left(1+\frac{t}{z}\right)^{\alpha+\beta-\frac{1}{2}} d t, \text { for } \beta-\alpha>-\frac{1}{2} .
$$

The confluent hypergeometric series of two variables is [G-R, p. 1031]

$$
\Phi_{1}(\alpha, \beta, \gamma, \mathrm{x}, \mathrm{y})=\sum_{\mathrm{m}, \mathrm{n}=0}^{\infty} \frac{(\alpha)_{\mathrm{m}+\mathrm{n}}(\beta)_{\mathrm{m}}}{(\gamma)_{\mathrm{m}+\mathrm{n}} \mathrm{m} ! \mathrm{n} !} \mathrm{x}^{\mathrm{m}} \mathrm{y}^{\mathrm{n}} ;|\mathrm{x}|<1 .
$$

The integral representation of (6) is [G-R, p. 349]

$$
\Phi_{1}(\alpha, \beta, \gamma, \mathrm{x}, \mathrm{y})=\frac{1}{\mathrm{~B}(\alpha, \gamma-\alpha)} \int_{0}^{1} \mathrm{t}^{\alpha-1}(1-\mathrm{t})^{\gamma-\alpha-1}(1-\mathrm{yt})^{-\beta} \mathrm{e}^{\mathrm{xt}} \mathrm{dt},
$$

for $\gamma>\alpha>0 \arg (1-\beta)<\pi$.

The hypergeometric functions of two variables is [G-R, p. 1018]

$$
\mathrm{F}_{1}\left[\alpha, \beta, \beta^{\prime}, \gamma ; \mathrm{x}, \mathrm{y}\right]=\sum_{\mathrm{m}=0}^{\infty} \sum_{\mathrm{n}=0}^{\infty} \frac{(\alpha)_{\mathrm{m}+\mathrm{n}}(\beta)_{\mathrm{m}}(\beta \prime)_{\mathrm{n}}}{(\gamma)_{\mathrm{m}+\mathrm{n}} \mathrm{m} ! \mathrm{n} !} \mathrm{x}^{\mathrm{m}} \mathrm{y}^{\mathrm{n}} ;|\mathrm{x}|<1,|\mathrm{y}|<1 .
$$

We also need the following important equations. 
For $u>0, m>0$, and $r>0$,

$$
\int_{u}^{\infty}(x+b)^{2 v-1}(x-u)^{2 r-1} e^{-m x} d x=\frac{(u+b)^{v+r-1}}{m^{v+r}} \exp \left[\frac{(b-u) m}{2}\right] \Gamma(2 r) W_{v-r, v+r-\frac{1}{2}}(u m+b m) .
$$

[G-R, p. 349]

For $\lambda>0, \mu>0$

$$
\int_{0}^{1} x^{\lambda-1}(1-x)^{\mu-1}(1-u x)^{-\varrho}(1-v x)^{-\sigma} d x=\mathrm{B}(\lambda, \mu) F_{1}[\lambda, \varrho, \sigma, \lambda+\mu ; u, v] .
$$

[G-R, p. 318]

The compound negative binomial distribution with exponential compounding distribution is defined as $X_{\rho}=\sum_{i=1}^{K} E_{i}$. The $E_{i} ; i=1,2, \ldots$ are independent and identically distributed with the exponential distribution having mean $\rho$, and $K$ is a negative binomial random variable with probability generating function $G_{K}(s)=\left(\rho /(1-(1-\rho) s)^{\alpha}\right.$. As usual, it is assumed that $\left\{E_{i}\right\}$ is independent of $K$. Then the moment generating function (mgf) of $X_{\rho}$ is

$$
\mathrm{M}_{\mathrm{X}}(\mathrm{t})=\left(\frac{\rho}{1-(1-\rho) \frac{1}{1-\rho \mathrm{t}}}\right)^{\alpha}=\left(\rho+(1-\rho) \frac{1}{1-\mathrm{t}}\right)^{\alpha} .
$$

The probability density function (pdf) of $X_{\rho}$ is

$$
\mathrm{f}(\mathrm{x})=\frac{1}{\Gamma(\alpha)} \frac{1-\rho}{\rho} \rho^{\alpha} \mathrm{e}^{-\frac{\mathrm{x}}{\rho}} \sum_{\mathrm{k}=0}^{\infty} \frac{\Gamma(\mathrm{k}+\alpha)}{\Gamma(\mathrm{k}) \mathrm{k} !}\left(\frac{1-\rho}{\rho} \mathrm{x}\right)^{\mathrm{k}-1} .
$$

Panjer and Willmot (1981) gave a version of the pdf with the finite sum. We denote this compound exponential negative binomial distribution by $\operatorname{CENB}(\alpha, \rho)$.

\section{Bivariate Gamma Distribution}

In the following, we list some bivariate gamma distributions. Without loss of generality, we consider bivariate gamma distribution with unit scale parameters, as we can always multiply each variable to get the required scale parameter.

\subsection{Double Gamma}

Let $Y_{i} \sim \Gamma\left(\alpha_{i}, 1\right), i=1,2$ be independent random variables. Then the joint pdf is

$$
\mathrm{f}_{\mathrm{Y}_{1}, \mathrm{Y}_{2}}\left(\mathrm{y}_{1}, \mathrm{y}_{2}\right)=\frac{1}{\Gamma\left(\alpha_{1}\right) \Gamma\left(\alpha_{2}\right)} \mathrm{y}_{1}^{\alpha_{1}-1} \mathrm{y}_{2}{ }^{\alpha_{2}-1} \exp \left(-\mathrm{y}_{1}-\mathrm{y}_{2}\right), \mathrm{y}_{1}>0, \mathrm{y}_{2}>0
$$

This distribution is known as double gamma distribution $D B \Gamma\left(\alpha_{1}, \alpha_{2}, 1,1\right)$.

The mgf is

$$
\mathrm{M}_{\mathrm{Y}_{1}, \mathrm{Y}_{2}}(\mathrm{t}, \mathrm{s})=\left(\frac{1}{1-\mathrm{t}}\right)^{\alpha_{1}}\left(\frac{1}{1-\mathrm{s}}\right)^{\alpha_{2}} .
$$

The convolution of double gamma distribution with another bivariate gamma distribution with equal scale parameters yields a new bivariate gamma distribution with same scale parameters and different shape parameters but with smaller correlation in absolute value.

\subsection{Complete Dependence Bivariate Gamma}

The complete dependence or degenerated bivariate gamma is defined as $Y_{1}=X_{0}$ and $Y_{2}=X_{0}$,

where $X_{0} \sim \Gamma\left(\alpha_{0}, 1\right)$. The distribution is degenerated in the line $\left\{\left(y_{1}, y_{2}\right): y_{1}=y_{2}\right\}$. The mgf is given by

$$
\mathrm{M}_{\mathrm{Y}_{1}, \mathrm{Y}_{2}}(\mathrm{t}, \mathrm{s})=\left(\frac{1}{1-\mathrm{t}-\mathrm{s}}\right)^{\alpha_{0}} .
$$

Obviously, the correlation between $Y_{1}$ and $Y_{2}$ is one.

If $\boldsymbol{U}$ is any bivariate gamma distribution, then its convolution with complete dependence gamma distribution after proper scaling will yield another bivariate gamma distribution with new scaling parameters. The covariance increases by $\operatorname{Var}\left(X_{0}\right)$. 


\subsection{Cheriyan's BivariateGamma}

Cheriyan (1941) derived a bivariate gamma using reduction method. Let $X_{i} \sim \Gamma\left(\alpha_{i}, 1\right), i=0,1,2$, be three independent random variables, where $Y_{i}=X_{0}+X_{i}, i=1,2$. The joint pdf is

$$
\mathrm{f}_{\mathrm{Y}_{1}, \mathrm{Y}_{2}}\left(\mathrm{y}_{1}, \mathrm{y}_{2}\right)=\frac{\exp \left(-\mathrm{y}_{1}-\mathrm{y}_{2}\right)}{\Gamma\left(\alpha_{1}\right) \Gamma\left(\alpha_{2}\right) \Gamma\left(\alpha_{0}\right)} \int_{0}^{\mathrm{y}_{1} \wedge \mathrm{y}_{2}}\left(\mathrm{y}_{1}-\mathrm{y}\right)^{\alpha_{1}-1}\left(\mathrm{y}_{2}-\mathrm{y}\right)^{\alpha_{2}-1} \mathrm{y}^{\alpha_{0}-1} \mathrm{e}^{-\mathrm{y}} \mathrm{dy}
$$

for $y_{1}>0, y_{2}>0$, and $\alpha_{i} \geq 0, i=0,1,2$.

The corresponding mgf is

$$
\mathrm{M}_{\mathrm{Y}_{1}, \mathrm{Y}_{2}}(\mathrm{t}, \mathrm{s})=\left(\frac{1}{1-\mathrm{t}}\right)^{\alpha_{1}}\left(\frac{1}{1-\mathrm{s}}\right)^{\alpha_{2}}\left(\frac{1}{1-\mathrm{t}-\mathrm{s}}\right)^{\alpha_{0}}
$$

The marginal distribution of $Y_{1}$ and $Y_{2}$ are gamma, with shape parameters $\alpha_{1}+\alpha_{0}, \alpha_{2}+\alpha_{0}$, and scale parameters 1 ,respectively.

Clearly, the distribution is the convolution of a double gamma distribution with a complete dependence bivariate gamma.

\subsection{Kibble’s BivariateGamma}

Kibble (1941) utilized the relation between normal distribution and gamma (chi-square) to introduced a bivariate gamma distribution

$$
\mathrm{f}_{\mathrm{Y}_{1}, \mathrm{Y}_{2}}\left(\mathrm{y}_{1}, \mathrm{y}_{2}\right)=\frac{(1-\rho)^{-\alpha}}{\Gamma(\alpha)} \sum_{\mathrm{j}=0}^{\infty} \mathrm{a}_{\mathrm{j}}\left(\mathrm{y}_{1} \mathrm{y}_{2}\right)^{\alpha+j-1} \exp \left(-\frac{\mathrm{y}_{1}+\mathrm{y}_{2}}{1-\rho}\right)
$$

for $y_{1}>0, y_{2}>0, \alpha>0$ and $0<\rho<1$, where $a_{j}=\frac{1}{\Gamma(\alpha+j) j !} \frac{\rho^{j}}{(1-\rho)^{2 j}}$.

The corresponding mgf is

$$
\mathrm{M}_{\mathrm{Y}_{1}, \mathrm{Y}_{2}}(\mathrm{t}, \mathrm{s})=\left(\frac{1}{1-\mathrm{t}-\mathrm{s}+(1-\rho) \mathrm{ts}}\right)^{\alpha}
$$

This distribution can be used to model the summer and winter streamflows and many other applications.

The marginal distributions of $Y_{1}$ and $Y_{2}$ are gamma with the same shape parameter $\alpha$ and scale parameter 1 . Here $\rho$ represents the correlation coefficient of the distribution. The special cases where $\rho=0$ and $\rho=1$ to correspond the double gamma distribution with equal shape parameters and the complete dependence gamma distribution, respectively. In the following, we give an alternative derivation of this distribution.

Theorem 1. Let $Y_{1}$ and $Y_{2}$ be two random variables defined as $Y_{i}=U_{i}+V_{i}, i=1,2$, where $U_{i}$ 's are iid $\Gamma\left(\alpha, \frac{1}{(1-\rho)}\right)$ and $V_{i}=\sum_{j=1}^{K} E_{i j}$, where $E_{i j}, i=1,2$, and $j=1,2, \ldots, K$ are iid random variables with exponential distribution function $\operatorname{Exp}\left(\frac{1}{1-\rho}\right)$, and $K \sim N B(\alpha, 1-\rho)$ is independent of $E_{i j}$ 's. Then $\left(Y_{1}, Y_{2}\right)$ has Kibble's bivariate gamma. The special case for $\alpha=1$ gives Moran-Downton bivariate exponential distribution introduced by Moran (1967) and Downton (1970).

Proof.

$$
\begin{gathered}
M_{Y_{1}, Y_{2}}(t, s)=E\left[e^{t\left(V_{1}+U_{1}\right)+s\left(V_{2}+U_{2}\right)}\right]=E\left[e^{t U_{1}+s U_{2}}\right] E\left[E\left(e^{t V_{1}+s V_{2}} \mid K\right)\right] \\
=\left(\frac{1}{1-(1-\rho) t}\right)^{\alpha}\left(\frac{1}{1-(1-\rho) s}\right)^{\alpha} E\left[\left(\left(\frac{1}{1-(1-\rho) t}\right)\left(\frac{1}{1-(1-\rho) s}\right)\right)^{K}\right] \\
=\left(\frac{1}{1-(1-\rho) t}\right)^{\alpha}\left(\frac{1}{1-(1-\rho) s}\right)^{\alpha}\left(\frac{1-\rho}{1-\rho\left(\frac{1}{1-(1-\rho) t}\right)\left(\frac{1}{1-(1-\rho) s}\right)}\right)^{\alpha}
\end{gathered}
$$




$$
=\left(\frac{1}{1-t-s+(1-\rho) t s}\right)^{\alpha} .
$$

A rescaled version of Kibble bivariate gamma distribution is the following

$$
f_{Y_{1}, Y_{2}}\left(y_{1}, y_{2}\right)=\frac{(1-\rho)^{-\alpha}}{\Gamma(\alpha)} \sum_{j=0}^{\infty} \frac{a_{j}}{\left(\lambda_{1} \lambda_{2}\right)^{\alpha+j}}\left(y_{1} y_{2}\right)^{\alpha+j-1} e^{-\left(\frac{1}{1-\rho}\right)\left(\frac{y_{1}}{\lambda_{1}}+\frac{y_{2}}{\lambda_{2}}\right)} .
$$

for $y_{1}>0, y_{2}>0, \alpha, \lambda_{1}, \lambda_{2}>0$ and $0<\rho<1$, where $a_{j}=\frac{1}{\Gamma(\alpha+j) j !} \frac{\rho^{j}}{(1-\rho)^{2 j}}$.

The corresponding mgf is

$$
\mathrm{M}_{\mathrm{Y}_{1}, \mathrm{Y}_{2}}(\mathrm{t}, \mathrm{s})=\left(\frac{1}{1-\lambda_{1} \mathrm{t}-\lambda_{2} \mathrm{~s}+\lambda_{1} \lambda_{2}(1-\rho) \mathrm{ts}}\right)^{\alpha} .
$$

\subsection{Gunst and Webster's BivariateGamma}

Gunst and Webster (1973) considered Jensen's bivariate gamma distribution when the scales parameters are equal. The joint pdf is

$$
\mathrm{f}_{\mathrm{Y}_{1}, \mathrm{Y}_{2}}\left(\mathrm{y}_{1}, \mathrm{y}_{2}\right)=\mathrm{y}_{1}{ }^{\alpha_{1}-1} \mathrm{y}_{2}{ }^{\alpha_{2}-1} \mathrm{e}^{-\left(\mathrm{y}_{1}+\mathrm{y}_{2}\right)} \sum_{\mathrm{j}=0}^{\infty} \frac{\Gamma(\alpha+\mathrm{j}) \rho_{\mathrm{j} !}}{\Gamma(\alpha) \Gamma\left(\alpha_{1}+\mathrm{j}\right) \Gamma\left(\alpha_{2}+\mathrm{j}\right)} \mathrm{L}_{\mathrm{j}}^{\left(\alpha_{1}-1\right)}\left(\mathrm{y}_{1}\right) \mathrm{L}_{\mathrm{j}}^{\left(\alpha_{2}-1\right)}\left(\mathrm{y}_{2}\right)
$$

where $L_{j}^{(\alpha)}(y)=\sum_{k=0}^{j}\left(\begin{array}{c}j+\alpha \\ j-k\end{array}\right) \frac{(-y)^{k}}{k !}$, for $y_{1}>0, y_{2}>0, \alpha_{1}>0, \alpha_{2}>0,0<\rho<1$, and $\alpha<\min \left(\alpha_{1}, \alpha_{2}\right)$.

The corresponding mgf is

$$
\mathrm{M}_{\mathrm{Y}_{1}, \mathrm{Y}_{2}}(\mathrm{t}, \mathrm{s})=\left(\frac{1}{1-\mathrm{t}}\right)^{\alpha_{1}}\left(\frac{1}{1-\mathrm{s}}\right)^{\alpha_{2}}\left(\frac{(1-\mathrm{t})(1-\mathrm{s})}{1-\mathrm{t}-\mathrm{s}+(1-\rho) \mathrm{ts}}\right)^{\alpha} .
$$

where $Y_{1}$ and $Y_{2}$ have marginal gamma distributions, with shape parameters $\alpha_{1}$ and $\alpha_{2}$ and scale parameters 1 , respectively.

Since $\alpha<\min \left(\alpha_{1}, \alpha_{2}\right)$, we can rewrite the mgf of Gunst and Webster's bivariate gamma as

$$
M_{Y_{1}, Y_{2}}(t, s)=\left(\frac{1}{1-t}\right)^{\alpha_{1}-\alpha}\left(\frac{1}{1-s}\right)^{\alpha_{2}-\alpha}\left(\frac{1}{1-t-s+(1-\rho) t s}\right)^{\alpha}
$$

Therefore, Gunst and Webster's bivariate gamma can be considered as Kibble's bivariate gamma convoluted with a double gamma distribution.

The above result motives a new bivariate gamma distribution as

$$
M_{Y_{1}, Y_{2}}(t, s)=\left(\frac{1}{1-t-s}\right)^{\beta}\left(\frac{1}{1-t-s+(1-\rho) t s}\right)^{\alpha} .
$$

The corresponding pdf is

$$
\begin{aligned}
f_{Y_{1}, Y_{2}}\left(y_{1}, y_{2}\right)= & \frac{(1-\rho)^{-\alpha}}{\Gamma(\alpha) \Gamma(\beta)}\left(y_{1} y_{2}\right)^{\alpha+j-1} e^{-\frac{y_{1}+y_{2}}{1-\rho}} \sum_{j=0}^{\infty} a_{j}\left(y_{1} \wedge y_{2}\right)^{\beta} \mathrm{B}(\beta, \alpha+j) \times \\
& \Phi_{1}\left(\beta, 1-\alpha-j, \alpha+\beta+j,\left(y_{1} \wedge y_{2}\right) \frac{1+\rho}{1-\rho},\left(\frac{y_{1}}{y_{2}}\right)^{\delta}\right) .
\end{aligned}
$$

where $\Phi_{1}(\alpha, \beta, \gamma, x, y)$ is the confluent hypergeometric (7) and $\delta=\left\{\begin{aligned} 1 & ; y_{1} \leq y_{2} \\ -1 & ; y_{1}>y_{2}\end{aligned}\right.$.

A rescaled version of Gunst and Webster's bivariate gamma distribution is the following

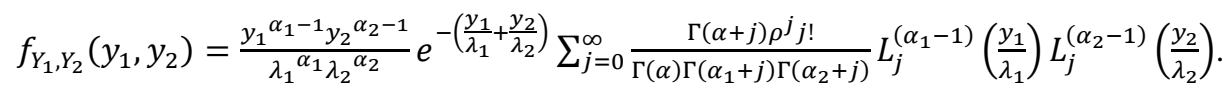


where $L_{j}^{(\alpha)}(y)=\sum_{k=0}^{j}\left(\begin{array}{l}j+\alpha \\ j-k\end{array}\right) \frac{(-y)^{k}}{k !}$, for $y_{1}>0, y_{2}>0, \alpha_{1}>0, \alpha_{2}>0, \lambda_{1}>0, \lambda_{2}>0,0<\rho<1$, and $\alpha<$ $\min \left(\alpha_{1}, \alpha_{2}\right)$.

The corresponding mgf is

$$
M_{Y_{1}, Y_{2}}(t, s)=\left(\frac{1}{1-\lambda_{1} t}\right)^{\alpha_{1}-\alpha}\left(\frac{1}{1-\lambda_{2} s}\right)^{\alpha_{2}-\alpha}\left(\frac{1}{1-\lambda_{1} t-\lambda_{2} s+(1-\rho) \lambda_{1} \lambda_{2} t s}\right)^{\alpha} .
$$

\subsection{Loaiciga and Leipnik's Bivariate Gamma}

Loaiciga and Leipnik (2005) derived a bivariate gamma of two correlated gamma variables. The joint pdf is

$$
f_{Y_{1}, Y_{2}}\left(y_{1}, y_{2}\right)=\sum_{n=0}^{\infty} \sum_{j=0}^{n} \sum_{k=0}^{n} A_{n j k}\left(y_{1}\right)^{\tau_{1}+j-n-1}\left(y_{2}\right)^{\tau_{2}+k-n-1} e^{-y_{1}-y_{2}},
$$

for $y_{1}>0, y_{2}>0$, where $\tau_{i}=\alpha_{i}(n+v), i=1,2$ and $A_{n j k}$ are given by

$$
A_{n j k}=\frac{(-1)^{n+j+k} \beta^{n}(n !)^{2}}{\Gamma\left(\tau_{1}\right) \Gamma\left(\tau_{2}\right) j ! k !}\left(\begin{array}{c}
-v \\
n
\end{array}\right)\left(\begin{array}{c}
\tau_{1}-1 \\
n-j
\end{array}\right)\left(\begin{array}{c}
\tau_{2}-1 \\
n-k
\end{array}\right) \text {, with } \alpha_{1}, \alpha_{2} \text {, and } v>0 \text {. }
$$

The mgf is

$$
M_{Y_{1}, Y_{2}}(t, s)=\left(\frac{1}{(1-t)^{\alpha_{1}(1-s)^{\alpha_{2}-\beta t s}}}\right)^{v}
$$

The marginal distribution of $Y_{1}$ and $Y_{2}$ are gamma, with shape parameters $v \alpha_{1}$ and $v \alpha_{2}$ and scale parameter 1 , respectively.

When $\alpha_{1}=\alpha_{2}=1$ the Loaiciga and Leipnik bivariate gamma distribution can be considered as Kibble's bivariate gamma.

\subsection{Nadarajah and Gupta sBivariateGamma}

Nadarajah and Gupta (2006) introduced two bivariate gamma distribution. These distributions are useful for modeling applications in several areas such as rainfall at two nearby rain gauges, wind gust data and the dependence between rainfall and runoff. The first one is defined as $Y_{1}=U X_{1}$ and $Y_{2}=U X_{2}$, where $\mathrm{U}$ has beta distribution with shape parameters $\alpha_{1}$ and $\alpha_{2} . X_{1}$ and $X_{2}$ are independent gamma distributions with common shape parameters $\alpha_{1}+\alpha_{2}$ and scale parameters 1 . Then $Y_{1}$ and $Y_{2}$ have a bivariate gamma distribution with joint pdf given by

$$
f_{Y_{1}, Y_{2}}\left(y_{1}, y_{2}\right)=C \frac{\left(y_{1} y_{2}\right)^{\alpha_{1}+\alpha_{2}-1}}{\left(y_{1}+y_{2}\right)^{\frac{\alpha_{1}+1}{2}+\alpha_{2}}} e^{-\left(\frac{y_{1}+y_{2}}{2}\right)} W_{\left(\frac{\alpha_{1}+1}{2}, \frac{\alpha_{1}+2 \alpha_{2}}{2}\right)}\left(y_{1}+y_{2}\right),
$$

where $C=\frac{1}{\Gamma\left(\alpha_{1}\right) \Gamma\left(\alpha_{1}+\alpha_{2}\right)}$, for $y_{1}>0, y_{2}>0$, and $\alpha_{1}, \alpha_{2}>0$.

The moment function is

$$
E\left(Y_{1}^{m} Y_{2}^{n}\right)=\frac{\Gamma\left(\alpha_{1}+m+n\right)}{\Gamma\left(\alpha_{1}\right)} \frac{\Gamma\left(\alpha_{1}+\alpha_{2}+m\right) \Gamma\left(\alpha_{1}+\alpha_{2}+n\right)}{\Gamma\left(\alpha_{1}+\alpha_{2}\right) \Gamma\left(\alpha_{1}+\alpha_{2}+m+n\right)} .
$$

The marginal distribution of $Y_{1}$ and $Y_{2}$ are gamma with common shape parameters $\alpha_{1}$ and scale parameter 1. The correlation coefficient is $\frac{\sqrt{\alpha_{1} \alpha_{2}}}{\alpha_{1}+\alpha_{2}+1}$.

Here, we derive the moment generating function as

Theorem 2. The mgf of Nadarajah and Gupta bivariate gamma distribution according to (30) is

$$
M_{Y_{1}, Y_{2}}(t, s)=F_{1}\left[\alpha_{1}, \alpha_{1}+\alpha_{2}, \alpha_{1}+\alpha_{2}, \alpha_{1}+\alpha_{2} ; t, s\right] .
$$


Proof.

$$
\begin{aligned}
& M_{Y_{1}, Y_{2}}(t, s)=E\left(e^{t\left(U X_{1}\right)+s\left(U X_{2}\right)}\right)=E\left(E\left(e^{U\left(t X_{1}+s X_{2}\right)} \mid U\right)\right)=E\left(\left(\frac{1}{1-t U} \frac{1}{1-s U}\right)^{\alpha_{1}+\alpha_{2}}\right)
\end{aligned}
$$

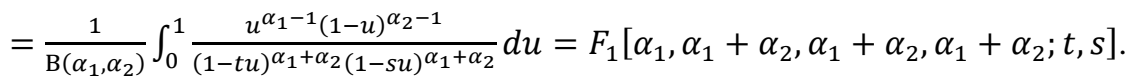

By using (8), where $F_{1}\left[\alpha, \beta, \beta^{\prime}, \gamma ; x, y\right]$ is the hypergeometric function (8).

The second Nadarajah and Gupta bivariate gamma distribution is defined as $Y_{1}=U_{1} X$ and $Y_{2}=U_{2} X$, where $\mathrm{X}$ has gamma distribution with shape parameter $\mathrm{c}$ and scale parameter 1 , where $a_{1}+b_{1}=a_{2}+b_{2}=c$. $U_{1}$ and $U_{2}$ are independent beta distributions with shape parameters $\left(a_{1}, b_{1}\right)$ and $\left(a_{2}, b_{2}\right)$, respectively. Then $Y_{1}$ and $Y_{2}$ have a bivariate gamma distribution with joint pdf given by

$$
\left.f_{Y_{1}, Y_{2}}\left(y_{1}, y_{2}\right)=C \sum_{j=0}^{\infty} \frac{(-1)^{j} \Gamma\left(b_{1}\right) \Gamma\left(b_{2}\right) e^{-\frac{y_{1}}{2}}}{j ! \Gamma\left(b_{2}-j\right)} y_{1}^{\frac{a_{1}+b_{2}-j-3}{2}} y_{2}{ }^{a_{2}+j-1} W_{\left(\frac{b_{2}-b_{1}-c-j-1}{2}\right.}, \frac{b_{1}+b_{2}-c-j}{2}\right)\left(y_{1}\right),
$$

for $y_{1} \geq y_{2}>0, a_{1}, a_{2}, b_{1}, b_{2}>0$, and $a_{1}+b_{1}=a_{2}+b_{2}=c$, where the constant $\mathrm{C}$ is $\frac{1}{c}=\Gamma(c) \mathrm{B}\left(a_{1}, b_{1}\right) \mathrm{B}\left(a_{2}, b_{2}\right)$. The moment function is

$$
E\left(Y_{1}^{m} Y_{2}^{n}\right)=\frac{\Gamma(c+m+n) \mathrm{B}\left(a_{1}+m, b_{1}\right) \mathrm{B}\left(a_{2}+n, b_{2}\right)}{\Gamma(c) \mathrm{B}\left(a_{1}, b_{1}\right) \mathrm{B}\left(a_{2}, b_{2}\right)}
$$

The marginal distribution of $Y_{1}$, and $Y_{2}$ are gamma with shape parameters $a_{1}$, and $a_{2}$, and scale parameter 1 , respectively. The correlation coefficient is $\frac{\sqrt{a_{1} a_{2}}}{c}$.

Here, we derive the moment generating function as

Theorem 3. The mgf of Nadarajah and Gupta bivariate gamma distribution according to (33) is

$$
M_{Y_{1}, Y_{2}}(t, s)=\left(\frac{1}{1-\ln \left({ }_{1} F_{1}\left[a_{1} ; c ; t\right]_{1} F_{1}\left[a_{2} ; c ; s\right]\right)}\right)^{c} .
$$

Proof.

$$
\begin{aligned}
M_{Y_{1}, Y_{2}}(t, s)= & E\left(e^{t\left(U_{1} X\right)+s\left(U_{2} X\right)}\right)=E\left(E\left(e^{X\left(t U_{1}+s U_{2}\right)} \mid X\right)\right)=E\left[\left({ }_{1} F_{1}\left[a_{1} ; c ; t\right]_{1} F_{1}\left[a_{2} ; c ; s\right]\right)^{X}\right] \\
& \left.=\int_{0}^{\infty} \frac{x^{c-1}}{\Gamma(c)} e^{-x\left(1-\ln \left[{ }_{1} F_{1}\left[a_{1} ; c ; t\right]{ }_{1} F_{1}\left[a_{2} ; c ; s\right]\right]\right.}\right) d x=\left(\frac{1}{1-\ln \left[{ }_{1} F_{1}\left[a_{1} ; c ; t\right]{ }_{1} F_{1}\left[a_{2} ; c ; s\right]\right]}\right)^{c} .
\end{aligned}
$$

\section{Bivariate Gamma Distribution Based on Self-Decomposability}

Let $Y \sim \Gamma(\alpha, 1)$ then it is well known from the self-decomposability of gamma distribution that for every $0<\rho<1$ there exists two independent random variables $X \sim \Gamma(\alpha, 1)$ and $X_{\rho}$ such that $Y=\rho X+X_{\rho}$ where $X_{\rho} \sim C E N B(\alpha, \rho)$. This kind of decomposition of gamma distribution can be used to introduce two different bivariate gamma distributions. We denote these two distributions by SD1 and SD2.

\subsection{SD1 Bivariate Gamma Distribution}

Let $Y_{1}=\rho X_{1}+X_{\rho}$ and $Y_{2}=\rho X_{2}+X_{\rho}$, where $X_{1}$ and $X_{2}$ are independent $\Gamma(\alpha, 1)$ and independent of $X_{\rho} \sim \operatorname{CENB}(\alpha, \rho)$. Then clearly both $Y_{1}$ and $Y_{2}$ have $\Gamma(\alpha, 1)$ with $\operatorname{Cov}\left(Y_{1}, Y_{2}\right)=\operatorname{Var}\left(X_{\rho}\right)=\alpha\left(1-\rho^{2}\right)$ and hence $\operatorname{Corr}\left(Y_{1}, Y_{2}\right)=\left(1-\rho^{2}\right)$.

The joint mgf is

$$
M_{Y_{1}, Y_{2}}(t, s)=\left(\frac{1}{1-\rho t}\right)^{\alpha}\left(\frac{1}{1-\rho s}\right)^{\alpha}\left(\rho+(1-\rho) \frac{1}{1-t-s}\right)^{\alpha} .
$$

and the pdf of $Y_{1}$ and $Y_{2}$ is 


$$
f_{Y_{1}, Y_{2}}\left(y_{1}, y_{2}\right)=\sum_{k=0}^{\infty} \frac{(1-\rho)^{k}}{\rho^{\alpha+k}} \frac{\left(y_{1} y_{2}\right)^{\alpha-1}}{\Gamma(\alpha)^{2} k !}\left(y_{1} \wedge y_{2}\right)^{k} e^{-\frac{y_{1}+y_{2}}{\rho}} \phi_{1}\left(\alpha, 1-\alpha, \alpha+k, \frac{y_{1} \wedge y_{2}}{\rho},\left(\frac{y_{1}}{y_{2}}\right)^{\delta}\right) .
$$

where $\Phi_{1}(\alpha, \beta, \gamma, x, y)$ is the confluent hypergeometric (7) and $\delta=\left\{\begin{array}{l}1 ; y_{1} \leq y_{2} \\ -1 ; y_{1}>y_{2}\end{array}\right.$.

Proof. Appendix A.1.

\subsection{SD2 Bivariate Gamma Distribution}

Let $Y_{1}=\rho_{1} X+X_{\rho_{1}}$ and $Y_{2}=\rho_{2} X+X_{\rho_{2}}$, where $X, X_{\rho_{1}}$ and $X_{\rho_{2}}$ are independent $X \sim \Gamma(\alpha, 1)$ and $X_{\rho_{i}} \sim C E N B\left(\alpha, \rho_{i}\right)$, $i=1,2$. Then clearly both $Y_{1}$ and $Y_{2}$ have $\Gamma(\alpha, 1)$ with $\operatorname{Cov}\left(Y_{1}, Y_{2}\right)=\alpha \rho_{1} \rho_{2}$, and $\operatorname{Corr}\left(Y_{1}, Y_{2}\right)=\rho_{1} \rho_{2}$. The joint mgf is

$$
M_{Y_{1}, Y_{2}}(t, s)=\left(\frac{1}{1-\rho_{1} t-\rho_{2} s}\right)^{\alpha}\left(\rho_{1}+\left(1-\rho_{1}\right) \frac{1}{1-t}\right)^{\alpha}\left(\rho_{2}+\left(1-\rho_{2}\right) \frac{1}{1-s}\right)^{\alpha}
$$

and the joint pdf is

$$
\begin{aligned}
f_{Y_{1}, Y_{2}}\left(y_{1}, y_{2}\right)= & \sum_{k=0}^{\infty} \sum_{l=0}^{\infty} A_{k, l} y_{1}{ }^{k+\frac{1+\delta}{2} \alpha-1} y_{2}{ }^{l+\frac{1-\delta}{2} \alpha-1} e^{-\frac{y_{1}}{\rho_{1}}-\frac{y_{2}}{\rho_{2}}} \times \\
& \phi_{1}\left(\alpha, 1-l^{\frac{1+\delta}{2}}-k^{\frac{1-\delta}{2}}, \alpha+k^{\frac{1+\delta}{2}}+l^{\frac{1-\delta}{2}},\left(\frac{y_{1}}{\rho_{1}}\right)^{\frac{1+\delta}{2}}\left(\frac{y_{2}}{\rho_{2}}\right)^{\frac{1-\delta}{2}},\left(\frac{\rho_{2}}{\rho_{1}} \frac{y_{1}}{y_{2}}\right)^{\delta}\right),
\end{aligned}
$$

where $A_{k, l}=\frac{\Gamma(l+\alpha) \Gamma(k+\alpha)}{\Gamma(\alpha)^{3} \Gamma(l) \Gamma(k) l ! k !} \mathrm{B}(\alpha, k)^{\frac{1+\delta}{2}} \mathrm{~B}(\alpha, l)^{\frac{1-\delta}{2}} \frac{\left(1-\rho_{1}\right)^{k}}{\rho_{1}{ }^{k+\frac{1+\delta}{2} \alpha-\alpha}} \frac{\left(1-\rho_{2}\right)^{l}}{\rho_{2}{ }^{l+\frac{1-\delta}{2} \alpha-\alpha}}$, and $\delta=\left\{\begin{array}{l}1 ; \frac{y_{1}}{\rho_{1}} \leq \frac{y_{2}}{\rho_{2}} \\ -1 ; \frac{y_{1}}{\rho_{1}}>\frac{y_{2}}{\rho_{2}}\end{array}\right.$.

Proof. Appendix A.2.

Explicit expressions for the pdfs of $Z=Y_{1}-Y_{2}$ for these four distributions are derived in Section 4.

\section{Bilateral Gamma Distribution $(D \Gamma)$}

For fitting real-life data, it is essential to find appropriate distributions. In many practical situations, the data show a tendency of asymmetry and heavy-tailed distributions. Examples of such data are price movements in financial markets, input-output processes, the formation of sand dunes, communications, and formation of solar neutrinos, etc. For those issues, normal distributions often provide a poor fit. In recent years, alternative models based on the difference of two non-negative random variables such as gamma, Poisson and negative binomial were developed. In this section, we introduced several new gamma difference distributions constructed from the bivariate gamma distributions defined in Section 2 and Section 3.

\subsection{Bilateral Double Gamma}

The bilateral double gamma $(B D \Gamma)$ was introduced by Küchler and Tappe (2008a), such as $\mathrm{Z}=Y_{1}-Y_{2}$, where $Y_{1}$ and $Y_{2}$ are independent $\Gamma\left(\alpha_{i}, \lambda_{i}\right), i=1,2$. This distribution will be denoted by $B D \Gamma\left(\alpha_{1}, \alpha_{2}, \lambda_{1}, \lambda_{2}\right)$. The probability density function of $\mathrm{Z}$ is

$$
f(z)=\frac{\left(\lambda_{1}\right)^{\frac{\alpha_{2}-\alpha_{1}}{2}}\left(\lambda_{2}\right)^{\frac{\alpha_{1}-\alpha_{2}}{2}}}{\Gamma\left(\alpha_{1}\right)\left(\lambda_{1}+\lambda_{2}\right)^{\frac{\alpha_{1}+\alpha_{2}}{2}}} Z^{\frac{\alpha_{1}+\alpha_{2}}{2}-1} e^{-\frac{z}{2}\left(\frac{\lambda_{2}-\lambda_{1}}{\lambda_{1} \lambda_{2}}\right)} W_{\frac{\alpha_{1}-\alpha_{2}}{2}, \frac{\alpha_{1}+\alpha_{2}-1}{2}}\left(Z\left(\frac{\lambda_{1}+\lambda_{2}}{\lambda_{1} \lambda_{2}}\right)\right) ; \quad z \geq 0,
$$

and $f\left(-z ; \alpha_{2}, \alpha_{1}, \lambda_{2}, \lambda_{1}\right)=f\left(z ; \alpha_{1}, \alpha_{2}, \lambda_{1}, \lambda_{2}\right)$ for $z<0$,

where $\alpha_{1}>0, \alpha_{2}>0, \lambda_{1}, \lambda_{2}>0$.

This pdf can be rewritten as

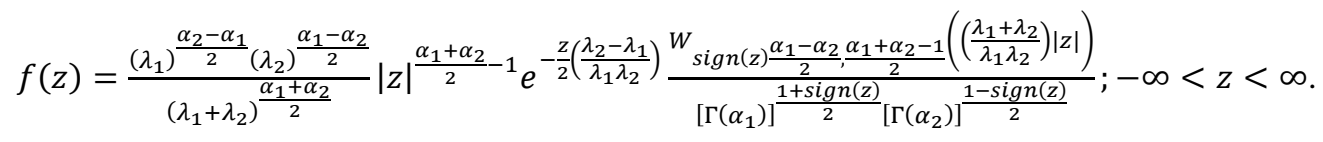


where $\operatorname{sign}(x)=\left\{\begin{array}{c}1 \text { for } x \geq 0 \\ -1 \text { for } x<0\end{array}\right.$.

The corresponding mgf is

$$
M_{Z}(t)=\left(\frac{1}{1-\lambda_{1} t}\right)^{\alpha_{1}}\left(\frac{1}{1+\lambda_{2} t}\right)^{\alpha_{2}} .
$$

The mean and the variance are $\mu=E(Z)=\alpha_{1} \lambda_{1}-\alpha_{2} \lambda_{2}$, and $\sigma^{2}=\operatorname{Var}(Z)=\alpha_{1} \lambda_{1}{ }^{2}+\alpha_{2} \lambda_{2}{ }^{2}$, respectively. For the special case where $\alpha_{1}=\alpha_{2}=\alpha$, we can write the $\mathrm{mgf}$ as

$$
M_{Z}(t)=\left(\frac{1}{1-\frac{\mu}{\alpha} t-\frac{\sigma^{2}-\mu^{2} / \alpha}{2 \alpha} t^{2}}\right)^{\alpha} .
$$

In the following proposition, a general form for the $\mathrm{n}^{\text {th }}$ moment is given

Theorem 4. If $Z$ has $B D \Gamma\left(\alpha_{1}, \alpha_{2}, \lambda_{1}, \lambda_{2}\right)$, then for $n \geq 1$

$$
E\left(Z^{n}\right)=\frac{\Gamma\left(\alpha_{1}+n\right)}{\Gamma \alpha_{1}} \lambda_{1}{ }_{2}{ }_{2} F_{1}\left(\alpha_{2},-n ; 1-\alpha_{1}-n ;-\frac{\lambda_{2}}{\lambda_{1}}\right),
$$

and

$$
E\left[(Z-\mu)^{n}\right]=\sum_{i=0}^{n}\left(\begin{array}{c}
n \\
i
\end{array}\right) \frac{\Gamma\left(\alpha_{1}+i\right)}{\Gamma \alpha_{1}} \lambda_{1}^{i}\left(\alpha_{2} \lambda_{2}-\alpha_{1} \lambda_{1}\right)^{n-i}{ }_{2} F_{1}\left(\alpha_{2},-i ; 1-\alpha_{1}-i ;-\frac{\lambda_{2}}{\lambda_{1}}\right) .
$$

Proof. Appendix B.1.

\subsection{Cheriyan’s Bilateral Gamma}

As the components of Cheriyan and Ramabhadran bivariate gamma are $Y_{1}=X_{0}+X_{1}$, and $Y_{2}=X_{0}+X_{2}$ then their difference is $Z=X_{1}-X_{2}$, which is the bilateral double gamma. Therefore Cheriyan's Bilateral Gamma is a bilateral double gamma.

\subsection{Kibble Bilateral Gamma}

Let the joint distribution of $Y_{1}$ and $Y_{2}$ be Kibble's bivariate gamma distribution, with parameters $\alpha, \lambda_{1}, \lambda_{2}>0$, and $0<\rho<1$. The Kibble bilateral gamma distribution is the distribution of the difference $\mathrm{Z}=Y_{1}-Y_{2}$. This distribution will be denoted by $B K \Gamma\left(\alpha, \lambda_{1}, \lambda_{2}, \rho\right)$. The probability density function is

$$
f_{Z}(z)=\sum_{j=0}^{\infty} b_{j} e^{\frac{-z}{2}\left(\frac{1}{1-\rho}\right)\left(\frac{1}{\lambda_{1}}-\frac{1}{\lambda_{2}}\right)}|z|^{\alpha+j-1} W_{0, \alpha+j-\frac{1}{2}}\left(|z|\left(\frac{1}{1-\rho}\right)\left(\frac{1}{\lambda_{1}}+\frac{1}{\lambda_{2}}\right)\right) ;-\infty<z<\infty .
$$

where $b_{j}=\frac{1}{\Gamma(\alpha) j !} \frac{\rho^{j}}{(1-\rho)^{j}} \frac{1}{\left(\lambda_{1}+\lambda_{2}\right)^{\alpha+j}}$.

The derivation of the pdf is in Appendix B.2.

The $\mathrm{mgf}$ is

$$
M_{Z}(t)=\left(\frac{1}{1-\left(\lambda_{1}-\lambda_{2}\right) t-\lambda_{1} \lambda_{2}(1-\rho) t^{2}}\right)^{\alpha} .
$$

Hence, the mean and the variance are $\mu=E(Z)=\alpha\left(\lambda_{1}-\lambda_{2}\right)$, and $\sigma^{2}=\alpha\left(\lambda_{1}-\lambda_{2}\right)^{2}+2 \alpha \lambda_{1} \lambda_{2}(1-\rho)$, respectively. In terms of the mean and the variance (47) can be written as

$$
M_{Z}(t)=\left(\frac{1}{1-\frac{\mu}{\alpha} t-\frac{\sigma^{2}-\mu^{2} / \alpha}{2 \alpha} t^{2}}\right)^{\alpha} .
$$

which is identical with (43). Thus the $B K \Gamma$ can be viewed as a special case of the $B D \Gamma$.

Remarks:

(i)The $B K \Gamma(\alpha, \lambda, \lambda, \rho)$ is identical with $B D \Gamma(\alpha, \alpha, \lambda \sqrt{1-\rho}, \lambda \sqrt{1-\rho})$.

(ii) The $B K \Gamma\left(\alpha, \lambda_{1}, \lambda_{2}, 0\right)$ is identical with $B D \Gamma\left(\alpha, \alpha, \lambda_{1}, \lambda_{2}\right)$.

(iii) For $\lambda_{1}>\lambda_{2}$, the $B K \Gamma\left(\alpha, \lambda_{1}, \lambda_{2}, 1\right)$ is $\Gamma\left(\alpha, \lambda_{1}-\lambda_{2}\right)$ while for $\lambda_{1}<\lambda_{2}$, the $B K \Gamma\left(\alpha, \lambda_{1}, \lambda_{2}, 1\right)$ is the negative of 
$\Gamma\left(\alpha, \lambda_{2}-\lambda_{1}\right)$.

\subsection{Gunst and Webster's Bilateral Gamma}

As Gunst and Webster's bivariate gamma is the convolution of $D B \Gamma$ and $K B \Gamma$, then the corresponding Gunst and Webster's bilateral gamma is $B D \Gamma\left(\alpha_{1}-\alpha, \alpha_{2}-\alpha, \lambda_{1}, \lambda_{2}\right)$ convoluted with $B K \Gamma\left(\alpha, \lambda_{1}, \lambda_{2}, \rho\right)$.

The $\mathrm{mgf}$ is

$$
M_{Z}(t)=\left(\frac{1}{1-\lambda_{1} t}\right)^{\alpha_{1}-\alpha}\left(\frac{1}{1+\lambda_{2} t}\right)^{\alpha_{2}-\alpha}\left(\frac{1}{1-\left(\lambda_{1}-\lambda_{2}\right) t-(1-\rho) \lambda_{1} \lambda_{2} t^{2}}\right)^{\alpha} .
$$

The pdf can be computed in term of Whittaker function but it will be more harder to deal with in term of inference.

\subsection{Loaiciga and Leipnik'sBilateral Gamma}

Let $Y_{1}$, and $Y_{2}$ follow Loaiciga and Leipnik's bivariate gamma distribution, with parameters $\alpha_{1}, \alpha_{2}, \alpha>0, \lambda_{1}, \lambda_{2}>0$, and $|\beta| \leq \lambda_{1} \lambda_{2} \sqrt{\alpha_{1} \alpha_{2}}$. The Loaiciga and Leipnikbilateral gamma distribution as $Z=Y_{1}-Y_{2}$. This distribution will be denoted by $B L \Gamma\left(\alpha, \alpha_{1}, \alpha_{2}, \beta, \lambda_{1}, \lambda_{2}\right)$. The pdf is

$$
f_{Z}(z)=\sum_{n=0}^{\infty} \sum_{j, k=0}^{n} B_{n j k}|z|^{\frac{\tau_{1}+\tau_{2}+j+k}{2}-n-1} \exp \left[\frac{-z}{2}\left(\frac{\lambda_{2}-\lambda_{1}}{\lambda_{1} \lambda_{2}}\right)\right] W_{\operatorname{sign}(z) \frac{\tau_{1}-\tau_{2}+j-k}{2}}, \frac{\tau_{1}+\tau_{2}+j+k-1}{2}-n\left(\left(\frac{\lambda_{1}+\lambda_{2}}{\lambda_{1} \lambda_{2}}\right)|z|\right),
$$

for $-\infty<z<\infty$, where $\tau_{i}=\alpha_{i}(n+\alpha)$ and $B_{n j k}$ are given by

$$
B_{n j k}=\frac{(-1)^{n+j+k} \beta^{n}(n !)^{2}\left(\frac{\lambda_{1} \lambda_{2}}{\lambda_{1}+\lambda_{2}}\right)^{\frac{\tau_{1}+\tau_{2}+j+k}{2}-n}}{\lambda_{1}{ }^{j+\tau_{1}} \lambda_{2}{ }^{{ }^{2} \tau_{2}} \Gamma\left(\tau_{1}\right) \Gamma\left(\tau_{2}\right) j ! k !}\left(\begin{array}{c}
-\alpha \\
n
\end{array}\right)\left(\begin{array}{c}
\tau_{1}-1 \\
n-j
\end{array}\right)\left(\begin{array}{c}
\tau_{2}-1 \\
n-k
\end{array}\right)\left[\Gamma\left(\tau_{2}+k-n\right)\right]^{\frac{1+\operatorname{sign}(z)}{2}}\left[\Gamma\left(\tau_{1}+j-n\right)\right]^{\frac{1-\operatorname{sign}(z)}{2}} .
$$

The derivation of the pdf is in Appendix B.3.

The mgf is

$$
M_{Z}(t)=\left(\frac{1}{\left(1-\lambda_{1} t\right)^{\alpha_{1}\left(1+\lambda_{2} t\right)^{\alpha_{2}}+\beta t^{2}}}\right)^{\alpha} .
$$

The mean and the variance are $E(Z)=\alpha\left(\alpha_{1} \lambda_{1}-\alpha_{2} \lambda_{2}\right)$, and $V(Z)=\alpha\left(\alpha_{1} \lambda_{1}{ }^{2}-\alpha_{2} \lambda_{2}{ }^{2}-2 \beta\right)$, respectively.

When $\alpha_{1}=\alpha_{2}=1$, The Loaiciga and Leipnik bilateral gamma is identical with $B K \Gamma\left(\alpha, \lambda_{1}, \lambda_{2}, \beta\right)$.

The triple summation with Whittaker function in the pdf makes the distribution more complicated for getting the properties and parameters estimation of the distribution.

\subsection{Nadarajah and Gupta'sBilateral Gamma}

The corresponding bilateral gamma distribution to Nadarajah and Gupta's bivariate gamma distribution has complicated pdf, therefore, it will not be discussed here.

\subsection{SD1 Bilateral Gamma}

Let $Y_{1}$, and $Y_{2}$ follow SD1 bivariate gamma distribution given in Section 3, and $\mathrm{Z}=Y_{1}-Y_{2}$, then $\mathrm{Z}$ has $B D \Gamma\left(\alpha, \alpha, \frac{1}{\rho}, \frac{1}{\rho}\right)$. So the SD1 bivariate gamma distribution does not provide a new difference distribution.

\subsection{SD2Bilateral Gamma}

Let $Y_{1}$ and $Y_{2}$ follow SD2 bivariate gamma distribution. The second self-decomposable bilateral gamma distribution as $\mathrm{Z}=Y_{1}-Y_{2}$. The $\mathrm{mgf}$ is

$$
M_{Z}(t)=\left(\frac{1}{1-\left(\rho_{1}-\rho_{2}\right) t}\right)^{\alpha}\left(\rho_{1} \rho_{2}+\rho_{2}\left(1-\rho_{1}\right) \frac{1}{1-t}+\rho_{1}\left(1-\rho_{2}\right) \frac{1}{1+t}+\left(1-\rho_{1}\right)\left(1-\rho_{2}\right) \frac{1}{1-t^{2}}\right)^{\alpha} .
$$

The mean and the variance are $E(Z)=0$, and $V(Z)=2 \alpha\left(1-\rho_{1} \rho_{2}\right)$, respectively.

The pdf is very complicated and involve triple summation with Whittaker function in the pdf makes the distribution more complicated for setting the properties and parameters estimation of the distribution.

\section{Conclusion}

Different bivariate gamma distributions provide different bilateral gamma distributions. However, under some conditions, 
one can see that bilateral gamma distributions are connected. By comparing the different bivariate gamma distributions, we found that Cheriyan and Ramabhadran bivariate gamma is the convolution of a double gamma distribution with a complete dependence bivariate gamma. Gunst and Webster's bivariate gamma can be considered as Kibble's bivariate gamma convoluted with double gamma distribution. Loaiciga and Leipnik's bivariate gamma is a generalization of Kibble's bivariate gamma.

The Kibble's bilateral gamma is a special case of the bilateral double gamma. Kibble's bilateral gamma is equivalent to Loaiciga and Leipnik bilateral gamma when $\alpha_{1}=\alpha_{2}=1$. Also, for $\lambda_{1}>\lambda_{2}$, the $B K \Gamma\left(\alpha, \lambda_{1}, \lambda_{2}, 1\right)$ is $\Gamma\left(\alpha, \lambda_{1}-\lambda_{2}\right)$ while for $\lambda_{1}<\lambda_{2}$, the $B K \Gamma\left(\alpha, \lambda_{1}, \lambda_{2}, 1\right)$ is the negative of $\Gamma\left(\alpha, \lambda_{2}-\lambda_{1}\right)$.

\section{Funding}

This project was funded by the National Plan for Science, Technology and Innovation (MAARIFAH), King Abdulaziz City for Science and Technology, Kingdom of Saudi Arabia, Award Number (11- MAT1856-02).

\section{References}

Balakrishnan, N.,\& Lai, C.D. (2009). Continuous Bivariate Distributions(2nd ed.). New York: Springer.

Becker, P.J.,\& Roux, J.J.J. (1981). A Bivariate Extension of the Gamma Distribution. South African Statistical Journal, 15, 1-12.

Bellini, F.,\& Mercuri, L. (2012). Option Pricing in a Conditional Bilateral Gamma model. [Working paper del dipartimento].https://doi.org/10.2139/ssrn.2046478

Cheng, J.,\& Berger, T. (2003). On the Difference of Two Sums of Independent Generalized Gamma Random Variables with Applications to Error Performance Analysis and Outage Probability Evaluation. IEEE International Symposium, Theory.https://doi.org/10.1109/ISIT.2003.1228053

Cheriyan, K.C. (1941). A Bivariate Correlated Gamma-Type Distribution Function. Journal of the Indian Mathematical Society 5, 133-144.

Coelho, C.A.,\& Mexia, J.T. (2007). On the Distribution of the Product and Ratio of Independent Generalized Gamma-Ratio Random Variables.Sankhya, 69, 221-255.

Downton, F. (1970). Bivariate Exponential Distributions in Reliability Theory. Journal of the Royal Statistical Society, Series $B, 32,408-417$.

Gunst, R.F.,\& Webster, J.T. (1973). Density Functions of the Bivariate Chi-Square Distribution. Journal of Statistical Computation and Simulation,2, 275-288.https://doi.org/10.1080/00949657308810052

Gradshteyn, I. S.,\& Ryzhik, I. M. (2007). Table of Integrals, Series, and Products(7th ed.). Burlington: Elsevier.

Holm, H.,\& Alouini, M. S. (2004). Sum and Difference of Two Squared Correlated Nakagami Variates in Connection With the McKay Distribution. IEEE Transactions on Communications, 52(8), 1367-1376.

Hutchinson, T.P.(1981). Compound Gamma Bivariate Distributions. Metrike, 28, 263-271.

Hutchinson, T.P.,\& Lai, C.D. (1990). Continuous Bivariate Distributions, Emphasising Applications. Adelaide, Australia: Rumsby Scientific Publishers.http://dx.doi.org/10.1093/acprof:oso/9780195309836.001.0001

Kibble, W.F. (1941). A Two-Variate Gamma Type Distribution. Sankhya,5, 137-150.

Küchler, U.,\& Tappe, S. (2008a) Bilateral Gamma Distributions and Processes in Financial Mathematics. Stochastic Processes and their Applications, 118(2), 261-283.https://doi.org/10.1016/j.spa.2007.04.006

Küchler, U.,\& Tappe, S. (2008b) On the Shapes of Bilateral Gamma Densities. Statistics and Probability Letters, 78(15), 2478-2484.https://doi.org/10.1016/j.spl.2008.02.039

Küchler, U.,\& Tappe, S. (2009) Option Pricing in Bilateral Gamma Stock Model. Statistics and Decisions, 27, $281-307$.

Loaiciga, H.A.,\& Leipnik, R.B. (2005). Correlated Gamma Variables in the Analysis of Microbial Densities in Water. Advances in Water Resources, 28, 329-335.https://doi.org/10.1016/j.advwatres.2004.11.008

Malik, H.J.,\& Trudel, R. (1985). Distributions of the Product and the Quotient from Bivariate t, F and Pareto Distributions. Communications in Statistics: Theory and Methods, 14, 2951-2962.

McKay, A.T. (1932). A Bessel Function Distribution. Biometrika,24. 39-44.

Moran, P.A.P. (1967). Testing for Correlation Between Non-Negative Variates. Biometrika,54, 385-394.

Nadarajah, S.,\& Gupta, A. K. (2006). Some Bivariate Gamma Distributions. Applied Mathematics Letters 19. $767-774$.

Panjer, H.H.,\& Willmot, G.E. (1981). Finite Sum Evaluation of the Negative Binomial- Exponential Model. Astin 
Bulletin, 12. 133-137.https://doi.org/10.1017/S0515036100007066

\section{Appendix A}

\section{SD1 Bivariate Gamma Distribution to Appendix A.1}

Let $Y_{1}=\rho X_{1}+X_{\rho}$ and $Y_{2}=\rho X_{2}+X_{\rho}$, where $X_{1}$ and $X_{2}$ are independent $\Gamma(\alpha, 1)$ and independent of $X_{\rho} \sim \operatorname{CENB}(\alpha, \rho)$.

The joint pdf of $Y_{1}$ and $Y_{2}$ is

$$
\begin{aligned}
& f\left(x_{1}, x_{2}, x_{\rho}\right)=\sum_{k=0}^{\infty} \frac{\Gamma(k+\alpha)}{\Gamma(\alpha)^{3} \Gamma(k) k !}\left(\frac{1-\rho}{\rho}\right)^{k} \rho^{\alpha} x_{1}{ }^{\alpha-1} x_{2}{ }^{\alpha-1} x_{\rho}{ }^{k-1} e^{-x_{1}-x_{2}-\frac{x \rho}{\rho}} \\
& f\left(y_{1}, y_{2}\right)=\sum_{k=0}^{\infty} \frac{\Gamma(k+\alpha)}{\Gamma(\alpha)^{3} \Gamma(k) k !}\left(\frac{1-\rho}{\rho}\right)^{k} \rho^{\alpha-2} e^{-\frac{y_{1}+y_{2}}{\rho}} \int_{0}^{\min \left(y_{1}, y_{2}\right)} x^{k-1}\left(\frac{y_{1}-x}{\rho}\right)^{\alpha-1}\left(\frac{y_{2}-x}{\rho}\right)^{\alpha-1} e^{\frac{x}{\rho}} d x \\
& =\sum_{k=0}^{\infty} \frac{\Gamma(k+\alpha)}{\Gamma(\alpha)^{3} \Gamma(k) k !} \frac{(1-\rho)^{k}}{\rho^{\alpha+k}} e^{-\frac{y_{1}+y_{2}}{\rho}} \int_{0}^{\min \left(y_{1}, y_{2}\right)} x^{k-1}\left(y_{1}-x\right)^{\alpha-1}\left(y_{2}-x\right)^{\alpha-1} e^{\frac{x}{\rho}} d x .
\end{aligned}
$$

For $y_{1} \leq y_{2}$

$$
\begin{gathered}
\int_{0}^{\min \left(y_{1}, y_{2}\right)} x^{k-1}\left(y_{1}-x\right)^{\alpha-1}\left(y_{2}-x\right)^{\alpha-1} e^{\frac{x}{\rho}} d x=\int_{0}^{y_{1}} x^{k-1}\left(y_{1}-x\right)^{\alpha-1}\left(y_{2}-x\right)^{\alpha-1} e^{\frac{x}{\rho}} d x \\
=y_{1}^{\alpha-1} y_{2}{ }^{\alpha-1} \int_{0}^{y_{1}} x^{k-1}\left(1-\frac{x}{y_{1}}\right)^{\alpha-1}\left(1-\frac{x}{y_{2}}\right)^{\alpha-1} e^{\frac{x}{\rho}} d x \\
=y_{1}{ }^{\alpha+k-1} y_{2}{ }^{\alpha-1} \int_{0}^{1} u^{k-1}(1-u)^{\alpha-1}\left(1-\frac{y_{1}}{y_{2}} u\right)^{\alpha-1} e^{\frac{y_{1}}{\rho} u} d u .
\end{gathered}
$$

By using (7)

$$
\int_{0}^{1} u^{k-1}(1-u)^{\alpha-1}\left(1-\frac{y_{1}}{y_{2}} u\right)^{\alpha-1} e^{\frac{y_{1}}{\rho} u} d u=B(k, \alpha) \phi_{1}\left(\alpha, 1-\alpha, \alpha+k, \frac{y_{1}}{\rho}, \frac{y_{1}}{y_{2}}\right)
$$

then,

$f\left(y_{1}, y_{2}\right)=\sum_{k=0}^{\infty} \frac{1}{\Gamma(\alpha)^{2} k !} \frac{(1-\rho)^{k}}{\rho^{\alpha+k}} y_{1}{ }^{\alpha+k-1} y_{2}{ }^{\alpha-1} e^{-\frac{y_{1}+y_{2}}{\rho}} \phi_{1}\left(\alpha, 1-\alpha, \alpha+k, \frac{y_{1}}{\rho}, \frac{y_{1}}{y_{2}}\right) ; y_{1}<y_{2}$.

For $y_{1}>y_{2}$, by the same above way.

\section{SD2 Bivariate Gamma Distribution to Appendix A.2}

Let $Y_{1}=\rho_{1} X+X_{\rho_{1}}$ and $Y_{2}=\rho_{2} X+X_{\rho_{2}}$, where $X, X_{\rho_{1}}$ and $X_{\rho_{2}}$ are independent $X \sim \Gamma(\alpha, 1)$ and $X_{\rho_{i}} \sim C E N B\left(\alpha, \rho_{i}\right)$, $i=1,2$. Then clearly both $Y_{1}$ and $Y_{2}$ have $\Gamma(\alpha, 1)$.

The joint pdf is

$$
\begin{aligned}
f\left(x_{\rho_{1}}, x_{\rho_{2}}, x\right)= & \sum_{k=0}^{\infty} \sum_{l=0}^{\infty} \frac{\Gamma(l+\alpha) \Gamma(k+\alpha)}{\Gamma(\alpha)^{3} \Gamma(l) \Gamma(k) l ! k !}\left(\rho_{1} \rho_{2}\right)^{\alpha}\left(\frac{1-\rho_{2}}{\rho_{2}}\right)^{l}\left(\frac{1-\rho_{1}}{\rho_{1}}\right)^{k} x^{\alpha-1} x_{\rho_{1}}{ }^{k-1} x_{\rho_{2}}{ }^{l-1} e^{-\frac{x \rho_{1}}{\rho_{1}}-\frac{x \rho_{2}}{\rho_{2}}-x} \\
f_{Y_{1}, Y_{2}}\left(y_{1}, y_{2}\right)= & \sum_{k=0}^{\infty} \sum_{l=0}^{\infty} \frac{\Gamma(l+\alpha) \Gamma(k+\alpha)}{\Gamma(\alpha)^{3} \Gamma(l) \Gamma(k) l ! k !}\left(\frac{1-\rho_{2}}{\rho_{2}}\right)^{l}\left(\frac{1-\rho_{1}}{\rho_{1}}\right)^{k} \rho_{1}{ }^{\alpha+k-1} \rho_{2}{ }^{\alpha+l-1} e^{-\frac{y_{1}}{\rho_{1}}-\frac{y_{2}}{\rho_{2}}} \\
& \times \int_{0}^{\frac{y_{1}}{\rho_{1}} \wedge \frac{y_{2}}{\rho_{2}}} e^{x}\left(\frac{y_{1}}{\rho_{1}}-x\right)^{k-1}\left(\frac{y_{2}}{\rho_{2}}-x\right)^{l-1} x^{\alpha-1} d x
\end{aligned}
$$


For $\frac{y_{1}}{\rho_{1}} \leq \frac{y_{2}}{\rho_{2}}$

$$
\begin{array}{r}
\int_{0}^{\frac{y_{1}}{\rho_{1}} \wedge \frac{y_{2}}{\rho_{2}}} e^{x}\left(\frac{y_{1}}{\rho_{1}}-x\right)^{k-1}\left(\frac{y_{2}}{\rho_{2}}-x\right)^{l-1} x^{\alpha-1} d x=\int_{0}^{\frac{y_{1}}{\rho_{1}}} e^{x}\left(\frac{y_{1}}{\rho_{1}}-x\right)^{k-1}\left(\frac{y_{2}}{\rho_{2}}-x\right)^{l-1} x^{\alpha-1} d x \\
=\left(\frac{y_{1}}{\rho_{1}}\right)^{k-1}\left(\frac{y_{2}}{\rho_{2}}\right)^{l-1}\left(\frac{y_{1}}{\rho_{1}}\right)^{\alpha} \int_{0}^{1} u^{\alpha-1}(1-u)^{k-1}\left(1-\frac{\rho_{2}}{y_{2}} \frac{y_{1}}{\rho_{1}} u\right)^{l-1} e^{\frac{y_{1}}{\rho_{1}} u} d x
\end{array}
$$

By using (7)

$$
\int_{0}^{1} u^{\alpha-1}(1-u)^{k-1}\left(1-\frac{\rho_{2}}{y_{2}} \frac{y_{1}}{\rho_{1}} u\right)^{l-1} e^{\frac{y_{1}}{\rho_{1}} u} d x=\mathrm{B}(\alpha, k) \phi_{1}\left(\alpha, 1-l, \alpha+k, \frac{y_{1}}{\rho_{1}}, \frac{y_{1}}{\rho_{1}} \frac{\rho_{2}}{y_{2}}\right)
$$

$f_{Y_{1}, Y_{2}}\left(y_{1}, y_{2}\right)=\sum_{k=0}^{\infty} \sum_{l=0}^{\infty} \frac{\Gamma(l+\alpha)}{\Gamma(\alpha)^{2} \Gamma(l) \Gamma(k) l ! k !}\left(\frac{1-\rho_{2}}{\rho_{2}}\right)^{l}\left(\frac{1-\rho_{1}}{\rho_{1}}\right)^{k} \rho_{2}^{\alpha} e^{-\frac{y_{1}}{\rho_{1}}-\frac{y_{2}}{\rho_{2}}} y_{1}{ }^{\alpha+k-1} y_{2}^{l-1} \phi_{1}\left(\alpha, 1-l, \alpha+k, \frac{y_{1}}{\rho_{1}}, \frac{y_{1}}{\rho_{1}} \frac{\rho_{2}}{y_{2}}\right)$,

for $\frac{y_{1}}{\rho_{1}}>\frac{y_{2}}{\rho_{2}}$, by the same above way.

\section{Appendix B}

The $n^{\text {th }}$ Moment of Küchler and Tappe Bilateral Gamma Distribution to Appendix B.1

If $Z$ has Küchler and Tappe bilateral gamma distribution, then the $\mathrm{n}^{\text {th }}$ moment is

$$
\begin{aligned}
& E\left(Z^{n}\right)=E\left[\left(X_{1}-X_{2}\right)^{n}\right]=E\left[\sum_{i=0}^{n}\left(\begin{array}{c}
n \\
i
\end{array}\right) X_{1}^{n-i}(-1)^{i} X_{2}^{i}\right] \\
& =\sum_{i=0}^{n}\left(\begin{array}{c}
n \\
i
\end{array}\right)(-1)^{i} E\left(X_{1}^{n-i}\right) E\left(X_{2}^{i}\right)=\sum_{i=0}^{n}\left(\begin{array}{c}
n \\
i
\end{array}\right)(-1)^{i} \frac{\Gamma\left(\alpha_{1}+n-i\right)}{\Gamma\left(\alpha_{1}\right)} \lambda_{1}{ }^{n-i} \frac{\Gamma\left(\alpha_{2}+i\right)}{\Gamma\left(\alpha_{2}\right)} \lambda_{2}{ }^{i} \\
& =\frac{\lambda_{1}{ }^{n}}{\Gamma \alpha_{1} \Gamma \alpha_{2}} \sum_{i=0}^{n}\left(\begin{array}{c}
n \\
i
\end{array}\right)(-1)^{i} \Gamma\left(\alpha_{2}+i\right) \Gamma\left(\alpha_{1}+n-i\right) \lambda_{1}{ }^{-i} \lambda_{2}{ }^{i} \\
& =\frac{\Gamma\left(\alpha_{1}+n\right)}{\Gamma \alpha_{1}} \lambda_{1}{ }^{n}{ }_{2} F_{1}\left(\alpha_{2},-n ; 1-\alpha_{1}-n ;-\frac{\lambda_{2}}{\lambda_{1}}\right) . \\
& E\left[(Z-\mu)^{n}\right]=E\left[\sum_{i=0}^{n}\left(\begin{array}{c}
n \\
i
\end{array}\right) Z^{i}(-\mu)^{n-i}\right]=\sum_{i=0}^{n}\left(\begin{array}{c}
n \\
i
\end{array}\right)(-\mu)^{n-i} E\left(Z^{i}\right) \\
& =\sum_{i=0}^{n}\left(\begin{array}{c}
n \\
i
\end{array}\right) \frac{\Gamma\left(\alpha_{1}+i\right)}{\Gamma \alpha_{1}} \lambda_{1}{ }^{i}\left(\alpha_{2} \lambda_{2}-\alpha_{1} \lambda_{1}\right)^{n-i}{ }_{2} F_{1}\left(\alpha_{2},-i ; 1-\alpha_{1}-i ;-\frac{\lambda_{2}}{\lambda_{1}}\right) .
\end{aligned}
$$

\section{Kibble Bilateral Gamma to Appendix B.2}

Let $Y_{1}$ and $Y_{2}$ follow Kibble's bivariate gamma distribution, with parameters $\alpha, \lambda_{1}, \lambda_{2}>0$, and $0<\rho<1$. The Kibble bilateral gamma distribution as $\mathrm{Z}=Y_{1}-Y_{2}$. This distribution will be denoted by $B K \Gamma\left(\alpha, \lambda_{1}, \lambda_{2}, \rho\right)$.

$$
f(z)=\frac{(1-\rho)^{-\alpha}}{\Gamma(\alpha)} \sum_{j=0}^{\infty} \frac{a_{j}}{\left(\lambda_{1} \lambda_{2}\right)^{\alpha+j}} e^{\left(\frac{1}{1-\rho}\right) \frac{z}{\lambda_{2}}} \times \int_{0 \vee Z}^{\infty} y_{1}^{\alpha+j-1}\left(y_{1}-z\right)^{\alpha+j-1} e^{-y_{1}\left(\frac{1}{1-\rho}\right)\left(\frac{1}{\lambda_{1}}+\frac{1}{\lambda_{2}}\right)} d y_{1},
$$

where $a_{j}=\frac{1}{\Gamma(\alpha+j) j !} \frac{\rho^{j}}{(1-\rho)^{2 j}}$.

By using (9)

$$
\int_{z}^{\infty} y_{1}^{\alpha+j-1}\left(y_{1}-z\right)^{\alpha+j-1} e^{-y_{1}\left(\frac{1}{1-\rho}\right)\left(\frac{1}{\lambda_{1}}+\frac{1}{\lambda_{2}}\right)} d y_{1}
$$




$$
\begin{gathered}
=\frac{z^{\alpha+j-1} \Gamma(\alpha+j)}{\left(\frac{1}{1-\rho}\right)^{\alpha+j}\left(\frac{1}{\lambda_{1}}+\frac{1}{\lambda_{2}}\right)^{\alpha+j} e^{\frac{-z}{2}\left(\frac{1}{1-\rho}\right)\left(\frac{1}{\lambda_{1}}+\frac{1}{\lambda_{2}}\right)} W_{0, \alpha+j-\frac{1}{2}}\left(z\left(\frac{1}{1-\rho}\right)\left(\frac{1}{\lambda_{1}}+\frac{1}{\lambda_{2}}\right)\right)} \\
\int_{0}^{\infty} y_{1}^{\alpha+j-1}\left(y_{1}-z\right)^{\alpha+j-1} e^{-y_{1}\left(\frac{1}{1-\rho}\right)\left(\frac{1}{\lambda_{1}}+\frac{1}{\lambda_{2}}\right)} d y_{1} \\
=\frac{(-z)^{\alpha+j-1} \Gamma(\alpha+j)}{\left(\frac{1}{1-\rho}\right)^{\alpha+j}\left(\frac{1}{\lambda_{1}}+\frac{1}{\lambda_{2}}\right)^{\alpha+j} e^{\frac{-z}{2}\left(\frac{1}{1-\rho}\right)\left(\frac{1}{\lambda_{1}}+\frac{1}{\lambda_{2}}\right)} W_{0, \alpha+j-\frac{1}{2}}\left(-z\left(\frac{1}{1-\rho}\right)\left(\frac{1}{\lambda_{1}}+\frac{1}{\lambda_{2}}\right)\right)}
\end{gathered}
$$

Then,

$f_{Z}(z)=\sum_{j=0}^{\infty} b_{j} e^{\frac{-z}{2}\left(\frac{1}{1-\rho}\right)\left(\frac{1}{\lambda_{1}}-\frac{1}{\lambda_{2}}\right)}|z|^{\alpha+j-1} W_{0, \alpha+j-\frac{1}{2}}\left(|z|\left(\frac{1}{1-\rho}\right)\left(\frac{1}{\lambda_{1}}+\frac{1}{\lambda_{2}}\right)\right)$,

where $b_{j}=\frac{1}{\Gamma(\alpha) j !} \frac{\rho^{j}}{(1-\rho)^{j}} \frac{1}{\left(\lambda_{1}+\lambda_{2}\right)^{\alpha+j}}$.

\section{Loaiciga and Leipnik's Bilateral Gamma to Appendix B.3}

Let $Y_{1}$, and $Y_{2}$ follow Loaiciga and Leipnik's bivariate gamma distribution, with parameters $\alpha_{1}, \alpha_{2}, \alpha>0, \lambda_{1}, \lambda_{2}>0$, and $|\beta| \leq \lambda_{1} \lambda_{2} \sqrt{\alpha_{1} \alpha_{2}}$. The Loaiciga and Leipnikbilateral gamma distribution as $\mathrm{Z}=Y_{1}-Y_{2}$. The probability density function is

$$
f_{Z}(z)=\sum_{n=0}^{\infty} \sum_{j=0}^{n} \sum_{k=0}^{n} A_{n j k} \exp \left(+\frac{z}{\lambda_{2}}\right) \int_{0 \vee z}^{\infty}(x)^{\tau_{1}+j-n-1}(x-z)^{\tau_{2}+k-n-1} \exp \left(-x\left(\frac{1}{\lambda_{1}}+\frac{1}{\lambda_{2}}\right)\right) d x
$$

where $\tau_{i}=\alpha_{i}(n+v)$ and $A_{n j k}$ are given by

$$
A_{n j k}=\frac{(-1)^{n+j+k} \beta^{n}(n !)^{2}}{\lambda_{1}{ }^{j+\tau_{1}} \lambda_{2}{ }^{k+\tau_{2}} \Gamma\left(\tau_{1}\right) \Gamma\left(\tau_{2}\right)}\left(\begin{array}{c}
-v \\
n
\end{array}\right)\left(\begin{array}{c}
\tau_{1}-1 \\
n-j
\end{array}\right)\left(\begin{array}{c}
\tau_{2}-1 \\
n-k
\end{array}\right)
$$

By using (9)

$$
\begin{aligned}
\int_{z}^{\infty}(x)^{\tau_{1}+j-n-1}(x-z)^{\tau_{2}+k-n-1} \exp \left(-x\left(\frac{1}{\lambda_{1}}+\frac{1}{\lambda_{2}}\right)\right) d x \\
=\frac{z^{\frac{\tau_{1}+j+\tau_{2}+k-2 n}{2}-1}}{\left(\frac{1}{\lambda_{1}}+\frac{1}{\lambda_{2}}\right)^{\frac{\tau_{1}+j+\tau_{2}+k-2 n}{2}}} \exp \left[\frac{-z}{2}\left(\frac{1}{\lambda_{1}}+\frac{1}{\lambda_{2}}\right)\right] \Gamma\left(\tau_{2}+k-n\right) W_{\frac{\tau_{1}+j-\tau_{2}-k}{2}}, \frac{\tau_{1}+j+\tau_{2}+k-2 n-1}{2}\left(z\left(\frac{1}{\lambda_{1}}+\frac{1}{\lambda_{2}}\right)\right) \\
\int_{0}^{\infty}(x)^{\tau_{1}+j-n-1}(x-z)^{\tau_{2}+k-n-1} \exp \left(-x\left(\frac{1}{\lambda_{1}}+\frac{1}{\lambda_{2}}\right)\right) d x \\
=\frac{(-z)^{\frac{\tau_{1}+j+\tau_{2}+k-2 n}{2}}-1}{\left(\frac{1}{\lambda_{1}+\frac{1}{\lambda_{2}}}\right)} \exp \left[\frac{-z}{2}\left(\frac{1}{\lambda_{1}}+\frac{1}{\lambda_{2}}\right)\right] \Gamma\left(\tau_{1}+j-n\right) W_{-\frac{\tau_{1}+j-\tau_{2}-k}{2}}, \frac{\tau_{1}+j+\tau_{2}+k-2 n-1}{2}\left(-z\left(\frac{1}{\lambda_{1}}+\frac{1}{\lambda_{2}}\right)\right) .
\end{aligned}
$$

\section{Copyrights}

Copyright for this articleis retained by the author(s), with first publication rights granted to the journal.

This is an open-access article distributed under the terms and conditions of the CreativeCommons Attribution license (http://creativecommons.org/licenses/by/4.0/). 